\title{
UnMICST: Deep learning with real augmentation for robust segmentation of highly multiplexed images of human tissues
}

\section{Clarence Yapp}

Harvard Medical School https://orcid.org/0000-0003-1144-5710

\section{Edward Novikov}

Harvard Medical School

\section{Won-Dong Jang}

Harvard University

\section{Yu-An Chen}

Harvard Medical School

\section{Marcelo Cicconet}

Harvard Medical School

\section{Zoltan Maliga}

Harvard Medical School

\section{Connor Jacobson}

Harvard Medical School https://orcid.org/0000-0002-0299-3451

\section{Donglai Wei}

Harvard Universitiy

\section{Sandro Santagata}

Brigham and Women's Hospital/ Harvard Medical School https://orcid.org/0000-0002-7528-9668

Hanspeter Pfister

Harvard

Peter Sorger ( $\square$ Peter_Sorger@hms.harvard.edu )

Harvard Medical School https://orcid.org/0000-0002-3364-1838

\section{Article}

Keywords: UnMICST, image processing, highly multiplexed images, microscopy

Posted Date: June 21st, 2021

DOl: https://doi.org/10.21203/rs.3.rs-501324/v1 
License: (c) (i) This work is licensed under a Creative Commons Attribution 4.0 International License.

Read Full License

Additional Declarations: Yes there is potential Competing Interest. PKS is a member of the SAB or BOD member of Applied Biomath, RareCyte Inc., and Glencoe Software, which distributes a commercial version of the OMERO database; PKS is also a member of the NanoString SAB. In the last five years the Sorger lab has received research funding from Novartis and Merck. Sorger declares that none of these relationships have influenced the content of this manuscript. SS is a consultant for RareCyte Inc. The other authors declare no outside interests.

Version of Record: A version of this preprint was published at Communications Biology on November 18th, 2022. See the published version at https://doi.org/10.1038/s42003-022-04076-3. 


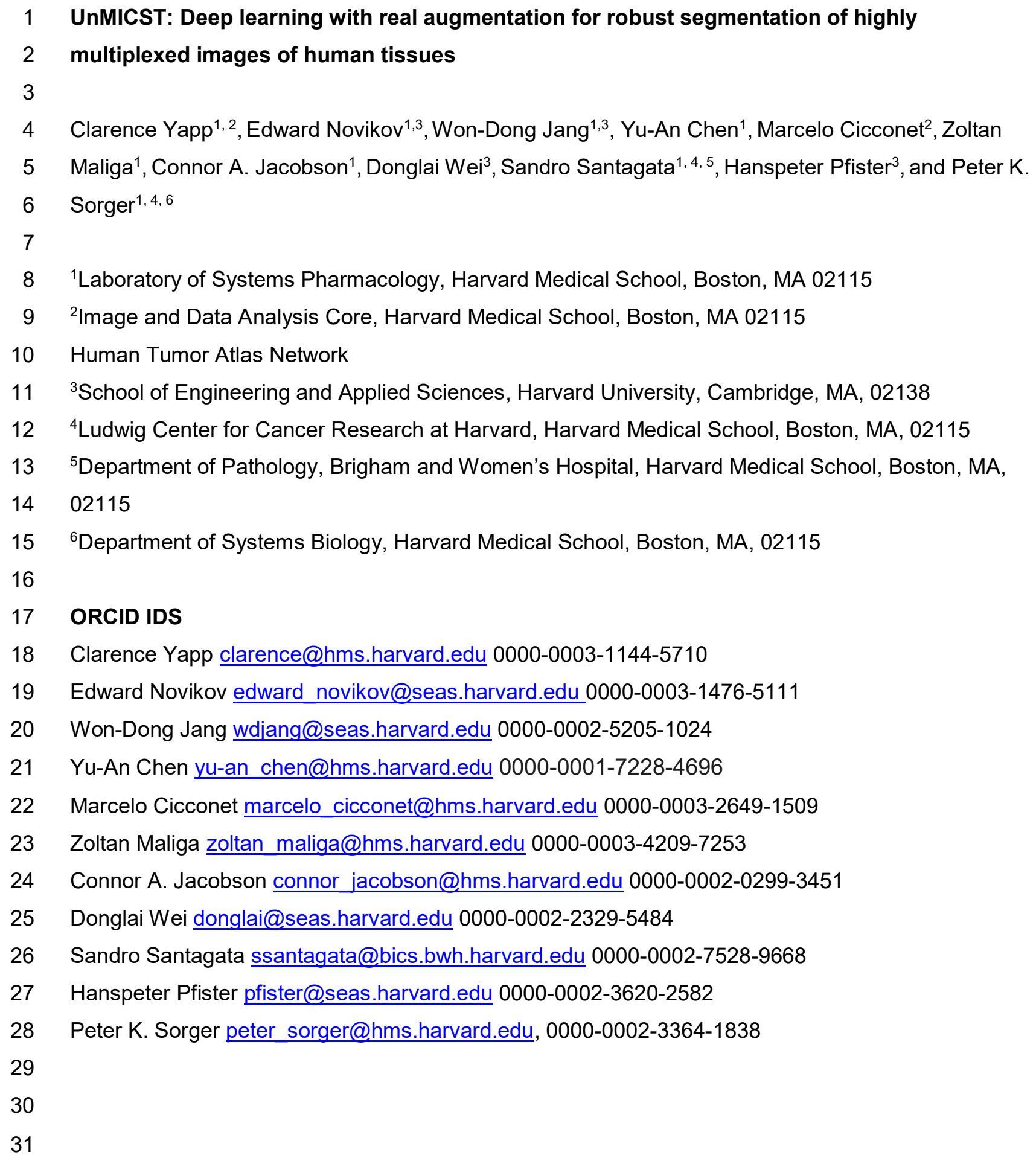


ABSTRACT

Newly developed technologies have made it feasible to routinely collect highly multiplexed (20-60 channel) images at subcellular resolution from human tissues for research and diagnostic purposes. Extracting single cell data from such images requires efficient and accurate image segmentation. This starts with identification of nuclei, a challenging problem in tissue imaging that has recently benefited from the use of deep learning. In this paper, we demonstrate two generally applicable approaches to improving segmentation accuracy for multiple human tissues. The first involves the use of "real augmentations" during training. These augmentations comprise defocused and saturated image data and improve model accuracy whereas computational augmentation (Gaussian blurring) does not. The second involves collection of nuclear envelope data to better identify nuclear outlines. The two approaches cumulatively and substantially improve segmentation with three different deep learning frameworks, yielding a set of highly accurate segmentation models. We speculate that the use of real augmentations may have applications in image processing outside of microscopy.

\section{INTRODUCTION}

Optical microscopy is a method of long-standing importance in biology that is changing rapidly due to the introduction of analytical methods based on machine learning (deep learning). Machine learning (ML) models are particularly important in the case of tissue images, whose complexity challenges traditional computer vision methods. Tissues are assemblies of multiple cell types that interact with each other and with structures such as basement membranes and connective tissue in defined geometric arrangements on length scales ranging from subcellular organelles to entire tissue $\left(0.1\right.$ to $\left.10^{4} \mu \mathrm{m}\right)$. Microscopy using Hematoxylin and Eosin (H\&E) complemented by immunohistochemistry ${ }^{1}$ has long played a primary role in the study of tissue architecture $^{2,3}$ and clinical histopathology remains the primary means by which diseases such as cancer are staged and managed clinically ${ }^{4}$. However, classical histology provides insufficient molecular information to precisely identify cell subtypes, study mechanisms of development, and characterize disease genes. In the past few years, several different methods for highly multiplexed tissue imaging have been developed (Supplementary Table 1). These yield subcellular resolution data on the abundance of 20-60 antigens, which is sufficient to subtype cells, measure cell states (quiescent, proliferating, dying, etc.) and interrogate cell signaling pathways. These methods differ in resolution, field of view, and multiplicity ("plex"), but all generate 2D images of tissue sections 5 $10 \mu \mathrm{m}$ thick. Developing effective image processing algorithms for these complex data is now the primary challenge in both research and clinical settings.

When multiplexed images are segmented and quantified, the resulting single cell data are a natural complement to single cell RNA Sequencing (scRNASeq) data, which have had a dramatic impact on our understanding of normal and diseased tissues ${ }^{5,6}$. Unlike dissociated RNASeq, 
however, multiplex tissue imaging preserves spatial information. Single cell analysis of data acquired via imaging requires segmentation, a computer vision technique that assigns class labels to an image in a pixel-wise manner to subdivide it; this is followed by marker quantification on a percell or per-organelle basis. Extensive work has gone into the development of methods for segmenting metazoan cells grown in culture, but segmentation of tissue images is a substantially more complex challenge. Recently, machine learning methods have become standard, paralleling the widespread use of convolutional neural networks (CNNs) in image recognition, object detection, and synthetic image generation ${ }^{7}$. Architectures such as ResNet, VGG16, and more recently, UNet and Mask R-CNN ${ }^{8,9}$ have gained attention for their ability to learn millions of parameters and generalize across datasets, as evidenced by excellent performance in a wide range of segmentation competitions, as well as in hackathon challenges ${ }^{10}$ using publicly available image datasets ${ }^{11,12}$. One limitation of machine learning on tissue images is a lack of sufficient freely-available data with ground truth labelling. Experience with natural scene images ${ }^{8}$ has proven that the acquisition of labels can be time consuming and rate limiting ${ }^{13}$.

In both cultured cells and tissues, the localization of nuclei is an optimal starting point for segmenting cells since most cell types have one nucleus (cells undergoing mitosis, muscle and liver cells and osteoclasts are important exceptions), and nuclear stains with high signal-to-background ratios are widely available. The nucleus is generally large relative to the resolution of wide-field fluorescence microscopes, making it easy to detect at multiple magnifications, and it often lies at the approximate center of a cell. There are possible advantages to using additional markers during image acquisition; for example, Schüffler et al., (2015) used multiplexed IMC data and watershed methods for multi-channel segmentation. Methods based on random forests such as llastik and Weka $^{15,16}$ also exploit multiple channels for class-wise pixel classification via an ensemble of decision trees to assign pixel-wise class probabilities in an image. However, random forest models have significantly less capacity for learning than CNNs, which is a substantial disadvantage. The possibility of using CNNs with multi-channel data to enhance nuclei segmentation has not been widely explored.

The accuracy of segmentation algorithms is crucially dependent on the quality of the original images and the absence of artefacts. In practice however, images have unavoidable blur artefacts and saturated channels. Segmentation methods must compensate for these aberrations. The standard way to accomplish this is via image augmentation ${ }^{17}$ which expands training sets by preprocessed images via random rotation, shearing, flipping, etc. This is designed to prevent algorithms from learning irrelevant aspects of an image, such as orientation. To date, focus artifacts have been tackled by using computed Gaussian blur to augment training data ${ }^{18-20}$. multiplexed tissue data by including different types of information in the images and by augmenting the training data. We generate a set of training and test data with ground-truth annotations via 
human curation of multiple normal tissues and tumors, and use these data to score segmentation accuracy achieved on three deep learning algorithms: UNet, Mask R-CNN, and Pyramid Scene Parsing Network (PSPNet); we use this to generate a family of Universal Models for Identifying Cells and Segmenting Tissue (UnMICST). We identify two generalizable and easily implemented ways to improve segmentation accuracy. The first involves adding images of nuclear envelope staining (NES) to images of nuclear chromatin acquired using DNA-intercalating dyes. The second involves adding real augmentations, defined here as intentionally defocused and over-saturated images (collected from the same specimens), to the training data to make models more robust to the types of artefacts encountered in real tissue images. We find that augmentation with real data significantly outperforms conventional Gaussian blur augmentation, offering a statistically significant improvement in model robustness.

\section{RESULTS}

Semantic segmentation is a coarse-grained ML approach that assigns objects to distinct trained classes, while instance segmentation is fine grained and identifies individual instances of objects. We implemented and evaluated two semantic and one instance segmentation algorithms (UNet, PSPNet, and Mask R-CNN respectively). We trained each of these models (UnMICST-U, UnMICST-P, and UnMICST-M, respectively) on manually curated and labelled data from seven distinct tissue types. The accuracy of each model was assessed based on the number of correctly segmented cells relative to the total number using a variable intersection over union (loU) threshold ranging from the least stringent, 0.55 , to most stringent, 0.8 . The loU (the Jaccard Index) is calculated by measuring the overlap between the ground truth annotation and the prediction via a ratio of the intersection to the union of pixels in both masks. The greater the loU, the higher the accuracy, with an ideal value of 1 (although this is very rarely achieved). Unlike the standard pixel accuracy metric (the fraction of pixels in an image that were correctly classified), loU is not sensitive to class-imbalance.

\section{Data sets and ground truth annotation of nuclear boundaries}

It is well established that various cell types in tissues have nuclear morphologies that are different from the spherical and ellipsoidal shape observed in cultured cells ${ }^{21}$. Nuclear pleomorphism (variation in nuclear size and shape) is widely used in histopathology to grade cancers and has been automated for H\&E images using classical machine vision methods ${ }^{22}$. We generated training, validation, and test datasets with manually annotated ground-truth of nuclei contours, centers, and background in images of a 120-core tissue microarray representing 30 distinct normal and diseased tissue types. Human inspection identified seven distinct nuclear morphologies ranging from mixtures of cells that were large vs. small, round cells vs. narrow, and densely and irregularly packed vs. organized in clusters. Because ground-truth annotation is a laborious process, we focused on 
distinctive nuclei from a subset of morphologies in seven tissue types (lung adenocarcinoma, nonneoplastic small intestine, normal prostate, colon adenocarcinoma, glioblastoma, non-neoplastic ovary, and tonsil) from 12 cores representing a total of $\sim 10,400$ nuclei.

\section{Real augmentations increase model robustness to focus artefacts}

To study the impact of real and computed augmentations on the performance of segmentation methods, we trained models with different sets of data, involving both real and computed augmentations and then tested the data on images that were acquired in focus, out of focus or blurred using a Gaussian kernel. We assessed segmentation accuracy quantitatively based on loU and qualitatively by visual inspection of predicted masks overlaid on image data. Real augmentation involved adding additional empirical, rather than computed, training data having the types of imperfections most commonly encountered in tissue. This was accomplished by positioning the focal plane $3 \mu \mathrm{m}$ above and below the specimen, resulting in de-focused images. A second set of images was collected at long exposure times, thereby saturating $70-80 \%$ of pixels. Because blurred and saturated images were collected sequentially without changing stage positions, it was possible to use the same set of ground truth annotations. For computed augmentations, we convolved a Gaussian kernel to the in-focus images with a range of standard deviations chosen to cover a broad spectrum of experimental cases (Figure 1a). In both scenarios, the resulting models were evaluated on a test set prepared in the same way as the training set.

In an initial set of studies, we found that models trained with data having Gaussian blur augmentation performed well on Gaussian blurred test data but when loU was quantified for defocused and saturated test data, we found that the use of training data augmented with Gaussian blur improved accuracy only slightly relative to baseline models lacking augmentations (Figure 1b). In contrast, the use of training data supplemented with real augmentations increased the fraction of cells retained at an loU threshold of 0.6 by $40-60 \%$; statistically significant improvement was observed up to an loU cutoff of 0.8 with all three learning frameworks (UnMICST-U, UnMICST-M, and UnMICST-P models). To perform a balanced comparison, we created two sets of training data having equal numbers of images. The first set contained the original data plus computed 90 - and 180-degree rotations, and the second set contained original data plus defocused data collected from above and below the specimen. Again, we found that models trained with real augmentations substantially outperformed rotationally augmented models when tested on defocused test data (Figure. 1c). Repeating this experiment with NES yielded the same outcome. Thus, training deep learning architectures with real augmentation generates models that outperform models with computed augmentation when data contain commonly encountered artefacts. 


\section{Addition of NES improves segmentation accuracy}

When we stained our TMA panel (the Exemplar Microscopy Images of Tissues and Tumors (EMIT) TMA) we found that antibodies against lamin A and C (Figure 2a) (which are different splice forms of $\angle M N A$ gene) stained approximately only half as many nuclei as antibodies against lamin $B 1$ (Figure 2b) or lamin B2 (Figure 2c) (products of the LMNB1 and LMNB2 genes). Staining for the lamin $B$ receptor (Figure 2e) exhibited poor image contrast. A pan-tissue survey showed that a mixture of antibodies for nucleoporin NUP98 and lamin B2 conjugated to the same fluorophore (Alexafluor-647) generated nuclear envelope staining (NES) for nearly all nuclei across multiple tissues (Figure 2f-h). We judged this to be the optimal antibody cocktail. However, only some cell types, epithelia in colorectal adenocarcinoma for example, exhibited the ring-like structure that is characteristic of nuclear lamina in cultured epithelial cells. The nuclear envelope in immune and other cells has folds and invaginations ${ }^{23}$ and in our data, NES staining could be irregular and diffuse, further emphasizing the difficulty of finding a broadly useful NES stain in tissue.

The value of NES images for model performance was also assessed quantitatively and qualitatively. In images of colon adenocarcinoma, non-neoplastic small intestine, and tonsil tissue, we found that the addition of NES images resulted in significant improvements in segmentation accuracy based on loU with all three learning frameworks; improvements in other tissues, such as lung adenocarcinoma, were more modest and sporadic (Figure 3a, Lung). For nuclear segmentation of fibroblasts in prostate cancer tissue, UnMICST-U and UnMICST-M models with NES data were no better than models trained on DNA staining alone. Most striking were cases in which NES data slightly decreased performance (UnMICST-P segmentation on prostrate fibroblasts and UnMICST-U segmentation of glioblastoma). Inspection of the UnMICST-P masks suggested that the segmentation of well-separated fibroblast nuclei was already optimal with DNA images alone ( $60 \%$ of nuclei retained at loU of 0.6$)$, implying the addition of NES images afforded little improvement. With UnMICST-U masks in glioblastoma, the problem appeared to involve atypical NES morphology, which is consistent with a high level of nuclear pleomorphism and the presence of "giant cells," both of which are well-established features of high-grade glioblastoma ${ }^{24,25}$. We also note that NES data alone was marginally inferior to DNA staining as a sole source of training data and should therefore be used in combination with images of DNA (Supplementary Figure 1). Thus, adding NES to training data broadly but not universally improves segmentation accuracy.

\section{Combining NES images and real augmentation has a cumulative effect}

To determine whether real augmentation and NES would combine during model training to achieve superior segmentation precision relative to the use of either type of data alone, we trained and tested models under four different scenarios (using all three learning frameworks; Figure 4). We used images from the small intestine, a tissue containing nuclei having a wide variety of morphologies, and then extended the analysis to other tissue types (see below). Models were 
217 evaluated on defocused DNA test data to increase the sensitivity of the experiment. In the first

218 scenario, we trained baseline models using in-focus DNA image data and tested models on unseen

219 in-focus DNA images. With tissues such as the small intestine, which are challenging to segment

220 because they contain densely-packed nuclei, scenario A resulted in slightly undersegmented

221 predictions. In Scenario $B$ and for all subsequent scenarios, defocused DNA images were included

222 in the test set, giving rise to contours that were substantially misaligned with ground truth

223 annotations and resulting in higher undersegmentation. False-positive predictions and imprecise

224 localizations of the nuclei membrane were observed in areas devoid of nuclei and with very low

225 contrast (Figure 4a). When NES images were included in the training set (Scenario C), nuclear

226 boundaries were more consistent with ground truth annotations, although false-positive predicted

227 nuclei still remained. The best performance was observed when NES images and real augmentation

228 were combined: accurate nuclear boundaries were well aligned with ground truth annotations in

229 both shape and in size. Observable differences in the placement of segmentation masks were

230 reflected in improvements in loU: for all three deep learning frameworks, including NES data and

231 real augmentations increased the fraction of nuclei retained by $50 \%$ at an loU threshold of 0.6

232 (Figure 4b). The accuracy of UnMICST-P (blue curve) trained on in-focus DNA data alone was

233 higher than the other two baseline models at all loU thresholds, suggesting that UnMICST-P has a

234 greater capacity to learn. UnMICST-P may have an advantage in experiments in which staining the

235 nuclear envelope proves difficult or impossible.

\section{Combining NES and real augmentation is advantageous across multiple tissue types}

238 To determine if improvements in segmentation would extend to multiple tissue types we repeated the analysis described above using three scenarios for training and testing with both in-

240 focus (Figure 5a) and defocused images (Figure 5b). Scenario 1 used in-focus DNA images for

241 training (blue bars), scenario 2 used in-focus DNA and NES images (red bars), and scenario 3 used

242 in-focus DNA and NES images plus real augmentation (green bars). While the magnitude of the

243 improvement varied with tissue type and test set (panel a vs b), the results as a whole support the

244 conclusion that including both NES and real augmentations during model training confers

245 statistically significant improvement in segmentation accuracy with multiple tissue types and models.

246 The accuracy boost was greatest when models performed poorly (e.g., in scenario 1 where models

247 were tested on defocused colon image data; Figure 5b, blue bars), so that segmentation accuracy

248 became relatively uniform across tissue and cell types.

250 Applying UnMICST to highly multiplex tissue images

To investigate the overall improvement achievable with UnMICST models, we tested them

252 with and without real or computed augmentations and NES data on all six tissues as a set, including 
in-focus, saturated and out-focus images (balancing the total amount of training data in each case).

254 A 1.7-fold improvement in accuracy was observed at an loU of 0.6 for the fully trained model (i.e.

255 with NES data and real augmentations; Figure 6a). Inspection of segmentation masks also

256 demonstrated more accurate contours for nuclei across a wide range of shapes. The overall

257 improvement in accuracy was substantially greater than any difference observed between semantic 258 and instance segmentation frameworks.

259 We then tested a fully trained UnMICST-U model on a 64-plex CyCIF image of non260 neoplastic small intestine tissue from the EMIT TMA (Figure 6b). Staining intensities were 261 quantified on a per-cell basis, and the results visualized using Uniform Manifold Approximation and

262 Projection (UMAP; Figure 6c). Segmentation masks were found to be well-located with little 263 evidence of under or over-segmentation (Figure $6 \mathrm{~d}$ ). Moreover, whereas $21 \%$ of cells with 264 segmented nuclei stained positive (as determined by using Gaussian-mixture model) for the 265 immune cell marker CD45, and 53\% stained positive for the epithelial cell marker E-cadherin, less 266 than 3\% were positive for both. No known cell type is actually positive for both CD45 and E267 cadherin, and the very low abundance of these double-positive "cells" is evidence of accurate 268 segmentation. When we examined some of the 830 double positive cells (blue dashed circle in 269 Figure 6c) we found multiple examples of a $\mathrm{CD}^{+} \mathrm{T}$ cell (yellow arrowheads; light yellow dots in 270 Figure 6e) tightly associated with or between the epithelial cells of intestinal villi (green "kiwi" 271 structure visible in Figure 6e). This is consistent with the known role of the intestinal epithelium in 272 immune homeostasis ${ }^{26}$. In these cases, the ability of humans to distinguish immune and epithelial 273 cells relies on prior knowledge, multi-dimensional intensity features and subtle differences in shape 274 and texture - none of which were aspects of model training. Thus, future improvements in tissue 275 segmentation are likely to require the development of CNNs able to classify rare but biologically 276 significant spatial arrangements, rather than simple extensions of the general purpose segmentation 277 algorithms described here.

\section{Some tissues still pose a challenge for nuclei segmentation}

Of all the tissue types annotated and tested in this paper, non-neoplastic ovary was the most difficult to segment (Supplementary Figure 2a) and addition of ovarian training data to models trained on data from other tissues decreased their accuracy (Supplementary Figure 2b). We have previously imaged ovarian cancers at even higher resolution (60x/1.42NA sampled at $108 \mathrm{~nm}$ pixel size; (Färkkilä et al., 2020) using optical sectioning and deconvolution microscopy; inspection of these images reveals nuclei with highly irregular morphology, poor image contrast, and dense packing (Supplementary Figure 2c) unlike colon adenocarcinoma (Supplementary Figure 2d).

287 Thus, additional research, possibly involving different NES antibodies, will be required to improve 288 performance with ovarian and other difficult to segment tissues. Until then, caution is warranted 289 when combining training data from tissues with very different nuclear morphologies. 
This paper makes three primary contributions to the growing literature on the segmentation of tissue images, which is an essential step in single-cell data analysis. First, we show that addition of real augmentations comprising defocused and saturated images to model training data improves segmentation accuracy to a significant extent whereas Gaussian blurring does not. These results extend to deep learning frameworks based on instance segmentation (UnMICST-M) and semantic segmentation (UnMICST-U and UnMICST-P). Second, we show that it is often possible to increase segmentation accuracy by including additional data (NES) on nuclear envelop morphology, although identifying suitable antibody cocktails is not trivial. Finally, using newly generated labeled training data for multiple tissue types, we show that real augmentation and NES combine to improve the robustness and accuracy of segmentation across many tissues; these improvements are directly applicable to the real-world task of segmenting high dimensional tissue and tumor images. UnMICST models are therefore a good starting point for performing image segmentation on rapidly growing tissue data repositories. Errors remaining when multiplexed images are segmented using optimized UnMICST models appear to have a subtle biological basis. Development of additional "physiology aware" machine-learning models may be necessary to reduce these apparent errors.

From a machine learning perspective, the value of adding images to training data is selfevident. Experimental feasibility is less clear. A key tradeoff is that the greater the number of fluorescence channels used for segmentation, the fewer channels available for the collection of data on other markers. Fortunately, the development of highly multiplexed imaging has made this less relevant because collection of 20-40 or more image channels (each corresponding to a different fluorescent antibody) has become routine. This makes it straightforward to reserve two channels for segmentation. The cost-benefit ratio of adding extra segmentation data will be different in high content screening of cells in multi-well plates, however, for which inexpensive reagents are generally essential. In tissues, lamins change in expression and morphology with disease state ${ }^{27}$ and imaging lamins is also likely to provide valuable biological information in addition to improving segmentation $^{28}$. To allow others to build on the current work, we are releasing all training and test images, their segmentation masks and annotations, and real augmentations for multiple types of tissue (tonsil, ovary, small intestine and cancers of the colon, brain, lung, prostate) via the EMIT resource; models are released as components of the UnMICST model resource (see data and code availability information).

The most immediately generalizable finding from this work is that real augmentation outperforms computed augmentation generated using Gaussian kernels. Blurring is an inevitable consequence of the limited bandwidth of optical systems (their point spread functions) as well as light scattering and diffraction, and in tissue imaging, the use of non-immersion objective lenses and consequent refractive index mismatches. Real out-of-focus blur also differs when the focal plane is above and below the specimen. Areas for future application of real augmentations could include 
inhomogeneous light sources and stage jitter. While it will be useful to determine kernels for more effective computed augmentation, collecting real augmentation data imposes a minimal burden in a real-world setting. Our observation that real augmentation outperforms computed augmentation may also have general significance outside of the field of microscopy: with any high-performance camera system, real out-of-focus data will inevitably be more complicated than Gaussian blur.

\section{CODE AND DATA AVAILABILITY}

To allow others to build on the current work we are releasing all training, validation and test images, their annotations, and real augmentations for multiple types of tissue (tonsil, ovary, small intestine and cancers of the colon, brain, lung, prostate) via the EMIT resource; models and scripts for training and inference are released as components of the UnMICST model resource (see data and code availability information). https://labsyspharm.github.io/UnMICST-info/

\section{METHODS}

\section{Sample preparation for imaging}

To generate images for model training and testing, human tissue specimens (from 42 patients) were used to construct a multi-tissue microarray (HTMA427) under an excess (discarded) tissue protocol approved by the Institutional Review Board (IRB) at Brigham and Women's Hospital (BWH IRB 2018P001627). Two $1.5 \mathrm{~mm}$ diameter cores were acquired from each of 60 tissue regions with the goal of acquiring one or two examples of as many tumors as possible (with matched normal tissue from the same resection when feasible), as well as several non-neoplastic medical diseases and secondary lymphoid tissues such as tonsil, spleen and lymph nodes. Overall, the tissue microarray (TMA) contained 120 cores plus three additional "marker cores," which are cores added to the TMA in a manner that makes it possible to orient the image. Slides were stained with the following reagents from Cell Signaling Technologies (Beverly MA, USA) and Abcam (Cambridge UK).

\begin{tabular}{|l|l|l|l|l|l|l|}
\hline Target & Fluorochrome & Species & Clone & Vendor & Cat. No. & RRID \\
\hline DNA & Hoechst 33342 & NA & NA & CST & 4082 & AB_10626776 \\
\hline Lamin B2 & Alexafluor 647 & Rabbit & EPR9701(B) & Abcam & ab200427 & AB_2889288 \\
\hline NUP98 & Alexafluor 647 & Rabbit & C39A3 & CST & 13393 & AB_2728831 \\
\hline
\end{tabular}
algorithmic evaluation, the images were split into three mutually disjoint subsets and used for training, validation, and testing. 


\section{Acquisition of image data and real augmentations}

The stained TMA was imaged on a INCell 6000 (General Electric Life Sciences) microscope equipped with a 20x/0.75 objective lens ( $370 \mathrm{~nm}$ nominal lateral resolution at $550 \mathrm{~nm}$ wavelength) and a pixel size of $0.325 \mu \mathrm{m}$ per pixel. Hoechst and lamin-A647 were excited with a $405 \mathrm{~nm}$ and $642 \mathrm{~nm}$ laser, respectively. Emission was collected with the "DAPI" (455/50 nm) and "Cy5" (682/60 $\mathrm{nm}$ ) filter sets with exposure times of $60 \mathrm{~ms}$ and $100 \mathrm{~ms}$, respectively. Whole-slide imaging involved acquisition of 1,215 tiles with an $8 \%$ overlap, which is recommended for stitching in ASHLAR, a next generation stitching and registration algorithm for large images (https://github.com/labsyspharm/ashlar). To generate defocused data, we acquired images from above and below the focal plane by varying the Z-axis by $3 \mu \mathrm{m}$ in both directions. To generate saturated images of DNA staining, a 150ms exposure time was used. These two types of "suboptimal" data were then used for "real augmentation" during model training, as described below.

Representative cores for lung adenocarcinoma, non-neoplastic small intestine, normal prostate, colon adenocarcinoma, glioblastoma, non-neoplastic ovary, and tonsil were extracted from image mosaics and down-sampled by a factor of 2 to match the pixel size of images routinely acquired and analyzed in $\mathrm{MCMICRO}^{29}$. Images were then cropped to 256 × 256-pixel tiles, and infocus DNA and NES were imported into Adobe Photoshop to facilitate human annotation of nuclear boundaries. Annotations for contours and background classes were labelled on separate layers while swapping between DNA and NES as necessary. To save time, we drew complete contours of nuclei and filled these in using the Matlab imfill operation to generate nuclei centers. For nuclei at the image borders where contours would be incomplete, we manually annotated nuclei centers. As described by Ronneberger et al. (2015), a fourth layer was used to mark areas between clumped cells. These additional annotations made it possible to specifically penalize models that incorrectly classified these pixels.

Because original, defocused, and saturated images of DNA were all acquired in the same image channels. To produce the training set, each image was cropped into $64 \times 64$ patches, normalized to use the full dynamic range, and further augmented using 90-degree rotations, reflections, and $20 \%$ upscaling. Consistent with the training set, the validation and test sets also include defocused and saturated examples but were not augmented with standard transformations. The ratio of data examples present in the training, validation, and test set split was 0.36:0.24:0.4. For a fair comparison across models, the same dataset and split were used for the three deep learning frameworks described in this manuscript (Supplementary Table 2).

\section{Model implementation}

To facilitate model training, three distinct state-of-the-art architectures were implemented and evaluated. They are, in no particular order, UNet, Mask R-CNN, and PSPNet. UNet was 
selected for its prior success in the biomedical domain, Mask R-CNN was selected for its ability to perform both object detection and mask generation, and PSPNet was selected for its capacity to integrate image features from multiple spatial scales. Training, validation, and test data were derived from 12 cores in 7 tissues and a total of 10,359 nuclei in the composition of colon -1,142; glioblastoma (GBM) - 675; lung - 1735; ovarian - 956; fibroblast - 922; small intestine - 1677; tonsil - 3252. To maintain consistency of evaluation across segmentation algorithms, segmentation accuracy was calculated by counting the fraction of cells in a held out test set that passed a sweeping Intersection over Union (IoU) threshold.

\section{Model Training}

\section{UnMICST-U models}

A three-class UNet model ${ }^{8}$ was trained based on annotation of nuclei centers, nuclei contours, and background. The neural network is comprised of 4 layers and 80 input features.

410 Training was performed using a batch size of 32 with the Adam Optimizer and a learning rate of 4110.00005 with a decay rate of 0.98 every 5,000 steps until there was no improvement in accuracy or $412 \sim 100$ epochs had been reached. Batch normalization was used to improve training speed. During 413 training, the bottom layer had a dropout rate of 0.35 , and $\mathrm{L} 1$ regularization was implemented to 414 minimize overfitting ${ }^{30,31}$ and early stopping. Training was performed on workstations equipped with 415 NVidia GTX 1080 or NVidia TitanX GPUs.

\section{UnMICST-M models}

Many segmentation models are based on the Mask R-CNN architecture ${ }^{9}$, Mask R-CNN has previously exhibited excellent performance on a variety of segmentation tasks. Mask R-CNN begins by detecting bounding boxes of nuclei and subsequently performs segmentation within each box. This approach eliminates the need for an intermediate watershed, or equivalent, segmentation step. Thus, Mask R-CNN directly calculates a segmentation mask, significantly reducing the overhead in traditional segmentation pipelines. We adopted a ResNet50 ${ }^{32}$ backbone model in the UnMICST-M implementation and initialized the weights using pretrained values from the COCO object instance segmentation challenge ${ }^{33}$ to improve convergence properties. For efficient training, we upsampled the original input images to $800 \times 800$-pixels and trained a model for 24 epochs using a batch size of 8 . The Adam optimizer, with a weight decay of 0.0001 to prevent overfitting, was exploited with a variable learning rate, initially set to 0.01 and decreased by a factor of 0.1 at epochs 16 and 22 . Training was performed on a compute node cluster using 4 NVidia TitanX or NVidia Tesla V100 GPUs. For evaluation and comparison, we used the model with the highest performance on the validation set, following standard practice. 
We trained a three class PSPNet model ${ }^{34}$ to extract cell nuclei centers, nuclei contours, and background from a wide variety of tissue types. PSPNet is one of the most widely used convolutional neural networks for the semantic segmentation of natural scene images in the computer vision field. The network employs a so-called pyramid pooling module whose purpose is to learn global as well as local features. The additional contextual information used by PSPNet allowed the segmentation algorithm to produce realistic probability maps with greater confidence. We used ResNet101 as a backbone. Training of the network was performed using a batch size of 8 with an image size of $256 \times 256$-pixels for 15,000 iterations or until the minimum loss model was obtained. A standard cross entropy loss function was used during training. Gradient descent was performed using the Adam optimizer with a learning rate of 0.0001 and a weight decay parameter of 0.005 via L2 regularization. Batch normalization was employed for faster convergence, and a dropout probability of 0.5 was used in the final network layer to mitigate overfitting. The model training was performed on a compute cluster node equipped with NVidia Tesla V100 GPUs.

\section{Analysis of multi-dimensional data}

For the analysis shown in Figure 6, a 64-plex CyCIF image of non-neoplastic small intestine tissue from the EMIT TMA (https://www.synapse.org/\#!Synapse:syn22345748/) was stained with a total of 45 antibodies as described in protocols https://www.protocols.io/view/ffpe-tissue-pretreatment-before-t-cycif-on-leica-bji2kkge and dx.doi.org/10.17504/protocols.io.bjiukkew. Images were segmented using the UnMICST-U model trained on DNA with NES data and real augmentations. Mean fluorescence intensities across 45 markers for 27,847 segmented nuclei were quantified as described in ${ }^{29}$. E-cadherin positive and CD45 positive cells were identified using Gaussian-mixture models on log-transformed data. For multivariate clustering, log-transformed mean intensities of all single cells of 14 selected protein markers (E-cadherin, pan-cytokeratin, CD45 CD4, CD3D, CD8, RF3, PML, GLUT1, GAPDH TDP43, OGT, COLL4, an EPCAM) were preprocessed using Uniform Manifold Approximation and Projection (UMAP) ${ }^{35}$ and clustered using Hierarchical Density-Based Spatial Clustering of Applications with Noise (HDBSCAN) ${ }^{36}$. Clusters expressing a high level of both E-cadherin and CD45 were identified and overlaid on a false-colored image showing the staining of DNA, E-cadherin, and CD45.

\section{ACKNOWLEDGEMENTS}

We thank Alyce Chen and Madison Tyler for their help with this manuscript. The work was funded by NIH grants U54-CA225088 and U2C-CA233262 to P.K.S. and S.S and by the Ludwig Cancer Center at Harvard. Z.M. is supported by NCI grant R50-CA252138. We thank Dana-Farber/Harvard 


\section{AUTHOR CONTRIBUTIONS}

472 The study design was conceived by CY, WDJ, EN, and PKS. Image acquisition and annotation was

473 done by CY. SS provided the EMIT TMA sample and validated the tissue types. TMA staining was

474 performed by ZM and CAJ. Data analysis was performed by CY, WDJ, EN, and YAC. YAC and CY

475 performed the single cell quantitative analysis and analysis found in figure 6. Additional coding was

476 done by MC. Additional experiments were conducted by DW. PKS, SS, HP supervised the study. All

477 authors contributed to the writing and editing of the manuscript.

478

\section{OUTSIDE INTERESTS}

480 PKS is a member of the SAB or BOD member of Applied Biomath, RareCyte Inc., and Glencoe

481 Software, which distributes a commercial version of the OMERO database; PKS is also a member

482 of the NanoString SAB. In the last five years the Sorger lab has received research funding from

483 Novartis and Merck. Sorger declares that none of these relationships have influenced the content of

484 this manuscript. SS is a consultant for RareCyte Inc. The other authors declare no outside interests. 
1. Immunologists, A. A. of. The Demonstration of Pneumococcal Antigen in Tissues by the Use of

$510 \quad$ Fluorescent Antibody. J. Immunol. 45, 159-170 (1942).

511 2. Albertson, D. G. Gene amplification in cancer. Trends Genet. 22, 447-455 (2006).

512 3. Shlien, A. \& Malkin, D. Copy number variations and cancer. Genome Med. 1, 62 (2009).

513 4. Amin, M. B. et al. The Eighth Edition AJCC Cancer Staging Manual: Continuing to build a bridge

514 from a population-based to a more 'personalized' approach to cancer staging. CA. Cancer J.

$515 \quad$ Clin. 67, 93-99 (2017).

516 5. Achim, K. et al. High-throughput spatial mapping of single-cell RNA-seq data to tissue of origin.

$517 \quad$ Nat. Biotechnol. 33, 503-509 (2015).

518 6. Slyper, M. et al. A single-cell and single-nucleus RNA-Seq toolbox for fresh and frozen human

519 tumors. Nat. Med. 26, 792-802 (2020).

520 7. LeCun, Y., Bengio, Y. \& Hinton, G. Deep learning. Nature 521, 436-444 (2015).

521 8. Ronneberger, O., Fischer, P. \& Brox, T. U-Net: Convolutional Networks for Biomedical Image 522 Segmentation. ArXiv150504597 Cs (2015).

523 9. He, K., Gkioxari, G., Dollár, P. \& Girshick, R. Mask R-CNN. ArXiv170306870 Cs (2018).

524 10. Caicedo, J. C. et al. Nucleus segmentation across imaging experiments: the 2018 Data Science

$525 \quad$ Bowl. Nat. Methods 16, 1247-1253 (2019).

526 11. Kromp, F. et al. An annotated fluorescence image dataset for training nuclear segmentation 527 methods. Sci. Data 7, 262 (2020).

528 12. Schwendy, M., Unger, R. E. \& Parekh, S. H. EVICAN—a balanced dataset for algorithm 529 development in cell and nucleus segmentation. Bioinformatics 36, 3863-3870 (2020).

530 13. Gurari, D. et al. How to Collect Segmentations for Biomedical Images? A Benchmark Evaluating 531 the Performance of Experts, Crowdsourced Non-experts, and Algorithms. 2015 IEEE Winter 532 Conf. Appl. Comput. Vis. (2015) doi:10.1109/WACV.2015.160.

533 14. Schüffler, P. J. et al. Automatic single cell segmentation on highly multiplexed tissue images. $534 \quad$ Cytometry A 87, 936-942 (2015). 
15. Arganda-Carreras, I. et al. Trainable Weka Segmentation: a machine learning tool for microscopy pixel classification. Bioinformatics 33, 2424-2426 (2017).

16. Berg, S. et al. ilastik: interactive machine learning for (bio)image analysis. Nat. Methods 16, 1226-1232 (2019).

17. Krizhevsky, A., Sutskever, I. \& Hinton, G. E. ImageNet Classification with Deep Convolutional Neural Networks. in Advances in Neural Information Processing Systems 25 (eds. Pereira, F., Burges, C. J. C., Bottou, L. \& Weinberger, K. Q.) 1097-1105 (Curran Associates, Inc., 2012).

18. Ahmed Raza, S. E. et al. MIMO-Net: A multi-input multi-output convolutional neural network for cell segmentation in fluorescence microscopy images. in 2017 IEEE 14th International Symposium on Biomedical Imaging (ISBI 2017) 337-340 (IEEE, 2017). doi:10.1109/ISBI.2017.7950532.

19. Shorten, C. \& Khoshgoftaar, T. M. A survey on Image Data Augmentation for Deep Learning. J. Big Data 6, 60 (2019).

20. Horwath, J. P., Zakharov, D. N., Mégret, R. \& Stach, E. A. Understanding important features of deep learning models for segmentation of high-resolution transmission electron microscopy images. Npj Comput. Mater. 6, 1-9 (2020).

21. Skinner, B. M. \& Johnson, E. E. P. Nuclear morphologies: their diversity and functional relevance. Chromosoma 126, 195-212 (2017).

22. Dalle, J.-R. et al. Nuclear pleomorphism scoring by selective cell nuclei detection. in IEEE Workshop on Applications of Computer Vision (WACV 2009), 7-8 December, 2009, Snowbird, UT, USA (IEEE Computer Society, 2009).

23. Fischer, E. G. Nuclear Morphology and the Biology of Cancer Cells. Acta Cytol. 64, 511-519 (2020).

24. Kros, J. M. Grading of Gliomas: The Road From Eminence to Evidence. J. Neuropathol. Exp. Neurol. 70, 101-109 (2011).

25. Louis, D., Ohgaki, H., Wiestler, O. \& Cavenee, W. WHO Classification of Tumours of the Central Nervous System. (2016). 
26. Allaire, J. M. et al. The Intestinal Epithelium: Central Coordinator of Mucosal Immunity. Trends Immunol. 39, 677-696 (2018).

27. Sakthivel, K. M. \& Sehgal, P. A Novel Role of Lamins from Genetic Disease to Cancer Biomarkers. Oncol. Rev. 10, 309 (2016).

28. Bell, E. S. \& Lammerding, J. Causes and consequences of nuclear envelope alterations in tumour progression. Eur. J. Cell Biol. 95, 449-464 (2016).

29. Schapiro, D. et al. MCMICRO: A scalable, modular image-processing pipeline for multiplexed tissue imaging. bioRxiv 2021.03.15.435473 (2021) doi:10.1101/2021.03.15.435473.

30. $\mathrm{Ng}, \mathrm{A}$. Y. Feature selection, L1 vs. L2 regularization, and rotational invariance. in Proceedings of the twenty-first international conference on Machine learning 78 (Association for Computing Machinery, 2004). doi:10.1145/1015330.1015435.

31. Srivastava, N., Hinton, G., Krizhevsky, A., Sutskever, I. \& Salakhutdinov, R. Dropout: a simple way to prevent neural networks from overfitting. J. Mach. Learn. Res. 15, 1929-1958 (2014).

32. He, K., Zhang, X., Ren, S. \& Sun, J. Deep Residual Learning for Image Recognition. in 2016 IEEE Conference on Computer Vision and Pattern Recognition (CVPR) 770-778 (2016). doi:10.1109/CVPR.2016.90.

33. Lin, T.-Y. et al. Microsoft COCO: Common Objects in Context. in Computer Vision - ECCV 2014 (eds. Fleet, D., Pajdla, T., Schiele, B. \& Tuytelaars, T.) 740-755 (Springer International Publishing, 2014). doi:10.1007/978-3-319-10602-1_48.

34. Zhao, H., Shi, J., Qi, X., Wang, X. \& Jia, J. Pyramid Scene Parsing Network. ArXiv161201105 Cs (2017).

35. Becht, E. et al. Dimensionality reduction for visualizing single-cell data using UMAP. Nat. Biotechnol. 37, 38-44 (2019).

36. Campello, R. J. G. B., Moulavi, D. \& Sander, J. Density-Based Clustering Based on Hierarchical Density Estimates. in Advances in Knowledge Discovery and Data Mining (eds. Pei, J., Tseng, V. S., Cao, L., Motoda, H. \& Xu, G.) 160-172 (Springer, 2013). doi:10.1007/978-3-642-37456- 
37. Otsu, N. A Threshold Selection Method from Gray-Level Histograms. (1979) doi:10.1109/TSMC.1979.4310076.

38. Byun, J. et al. Automated tool for the detection of cell nuclei in digital microscopic images: application to retinal images. Mol. Vis. 12, 949-960 (2006).

39. Xu, H., Lu, C. \& Mandal, M. An efficient technique for nuclei segmentation based on ellipse descriptor analysis and improved seed detection algorithm. IEEE J. Biomed. Health Inform. 18, 1729-1741 (2014).

40. Xu, H., Lu, C., Berendt, R., Jha, N. \& Mandal, M. Automatic Nuclei Detection Based on Generalized Laplacian of Gaussian Filters. IEEE J. Biomed. Health Inform. 21, 826-837 (2017).

41. Qi, X., Xing, F., Foran, D. J. \& Yang, L. Robust Segmentation of Overlapping Cells in

42. Liu, C., Shang, F., Ozolek, J. A. \& Rohde, G. K. Detecting and segmenting cell nuclei in twodimensional microscopy images. J. Pathol. Inform. 7, 42 (2016).

43. Lux, F. \& Matula, P. Cell Segmentation by Combining Marker-Controlled Watershed and Deep

45. Shahzad M et al. Robust Method for Semantic Segmentation of Whole-Slide Blood Cell

47. Al-Kofahi, Y., Zaltsman, A., Graves, R., Marshall, W. \& Rusu, M. A deep learning-based algorithm for 2-D cell segmentation in microscopy images. BMC Bioinformatics 19, 365 (2018). e2005970 (2018) 
49. Schmidt, U., Weigert, M., Broaddus, C. \& Myers, G. Cell Detection with Star-convex Polygons. ArXiv180603535 Cs 11071, 265-273 (2018).

50. Wen, C. et al. Deep-learning-based flexible pipeline for segmenting and tracking cells in 3D image time series for whole brain imaging. bioRxiv 385567 (2018) doi:10.1101/385567.

51. Vu, Q. D. et al. Methods for Segmentation and Classification of Digital Microscopy Tissue

53. Kromp, F. et al. Deep Learning architectures for generalized immunofluorescence based nuclear image segmentation. (2019).

54. Vuola, A. O., Akram, S. U. \& Kannala, J. Mask-RCNN and U-net Ensembled for Nuclei

52. Lugagne, J.-B., Lin, H. \& Dunlop, M. J. DeLTA: Automated cell segmentation, tracking, and lineage reconstruction using deep learning. PLOS Comput. Biol. 16, e1007673 (2020).

56. Liu, D. et al. Unsupervised Instance Segmentation in Microscopy Images via Panoptic Domain

59. Torr, A., Basaran, D., Sero, J., Rittscher, J. \& Sailem, H. DeepSplit: Segmentation of Microscopy Images Using Multi-task Convolutional Networks. in Medical Image Understanding and Analysis (eds. Papież, B. W., Namburete, A. I. L., Yaqub, M. \& Noble, J. A.) vol. 1248 155167 (Springer International Publishing, 2020). 
60. Stringer, C., Wang, T., Michaelos, M. \& Pachitariu, M. Cellpose: a generalist algorithm for cellular segmentation. Nat. Methods 1-7 (2020) doi:10.1038/s41592-020-01018-x.

61. Ljosa, V., Sokolnicki, K. L. \& Carpenter, A. E. Annotated high-throughput microscopy image sets for validation. Nat. Methods 9, 637 (2012).

62. Xing, F. et al. Towards pixel-to-pixel deep nucleus detection in microscopy images. BMC Bioinformatics 20, 472 (2019).

63. Yang, L. et al. NuSeT: A deep learning tool for reliably separating and analyzing crowded cells.

$$
\text { PLOS Comput. Biol. 16, e1008193 (2020). }
$$

64. Al-Kofahi, Y., Lassoued, W., Lee, W. \& Roysam, B. Improved Automatic Detection and Segmentation of Cell Nuclei in Histopathology Images. IEEE Trans. Biomed. Eng. 57, 841-852 (2010).

65. Roth, H. R. et al. DeepOrgan: Multi-level Deep Convolutional Networks for Automated Pancreas Segmentation. in Medical Image Computing and Computer-Assisted Intervention -- MICCAI 2015 (eds. Navab, N., Hornegger, J., Wells, W. M. \& Frangi, A.) 556-564 (Springer International Publishing, 2015). doi:10.1007/978-3-319-24553-9_68.

66. Chen, H., Qi, X., Yu, L. \& Heng, P.-A. DCAN: Deep Contour-Aware Networks for Accurate Gland Segmentation. ArXiv160402677 Cs (2016).

67. Höfener, H. et al. Deep learning nuclei detection: A simple approach can deliver state-of-the-art results. Comput. Med. Imaging Graph. 70, 43-52 (2018).

68. Khadangi, A., Boudier, T. \& Rajagopal, V. EM-net: Deep learning for electron microscopy image segmentation. bioRxiv 2020.02.03.933127 (2020) doi:10.1101/2020.02.03.933127.

69. Raza, S. E. A. et al. Micro-Net: A unified model for segmentation of various objects in microscopy images. Med. Image Anal. 52, 160-173 (2019).

70. Berryman, S., Matthews, K., Lee, J. H., Duffy, S. P. \& Ma, H. Image-based Cell Phenotyping Using Deep Learning. bioRxiv 817544 (2019) doi:10.1101/817544.

71. Challen, G. A. \& Goodell, M. A. Promiscuous Expression of H2B-GFP Transgene in Hematopoietic Stem Cells. PLOS ONE 3, e2357 (2008).

72. Tumbar, T. et al. Defining the epithelial stem cell niche in skin. Science 303, 359-363 (2004). 
673 73. Chalfoun, J. et al. FogBank: a single cell segmentation across multiple cell lines and image 674 modalities. BMC Bioinformatics 15, 431 (2014).

675 74. Stylianidou, S., Brennan, C., Nissen, S. B., Kuwada, N. J. \& Wiggins, P. A. SuperSegger: robust 676 image segmentation, analysis and lineage tracking of bacterial cells. Mol. Microbiol. 102, 690$677 \quad 700(2016)$.

678

679

680

681

682

683

684

685

686

687

688

689

690

691

692

693

694

695 


\section{Figure 1}

a

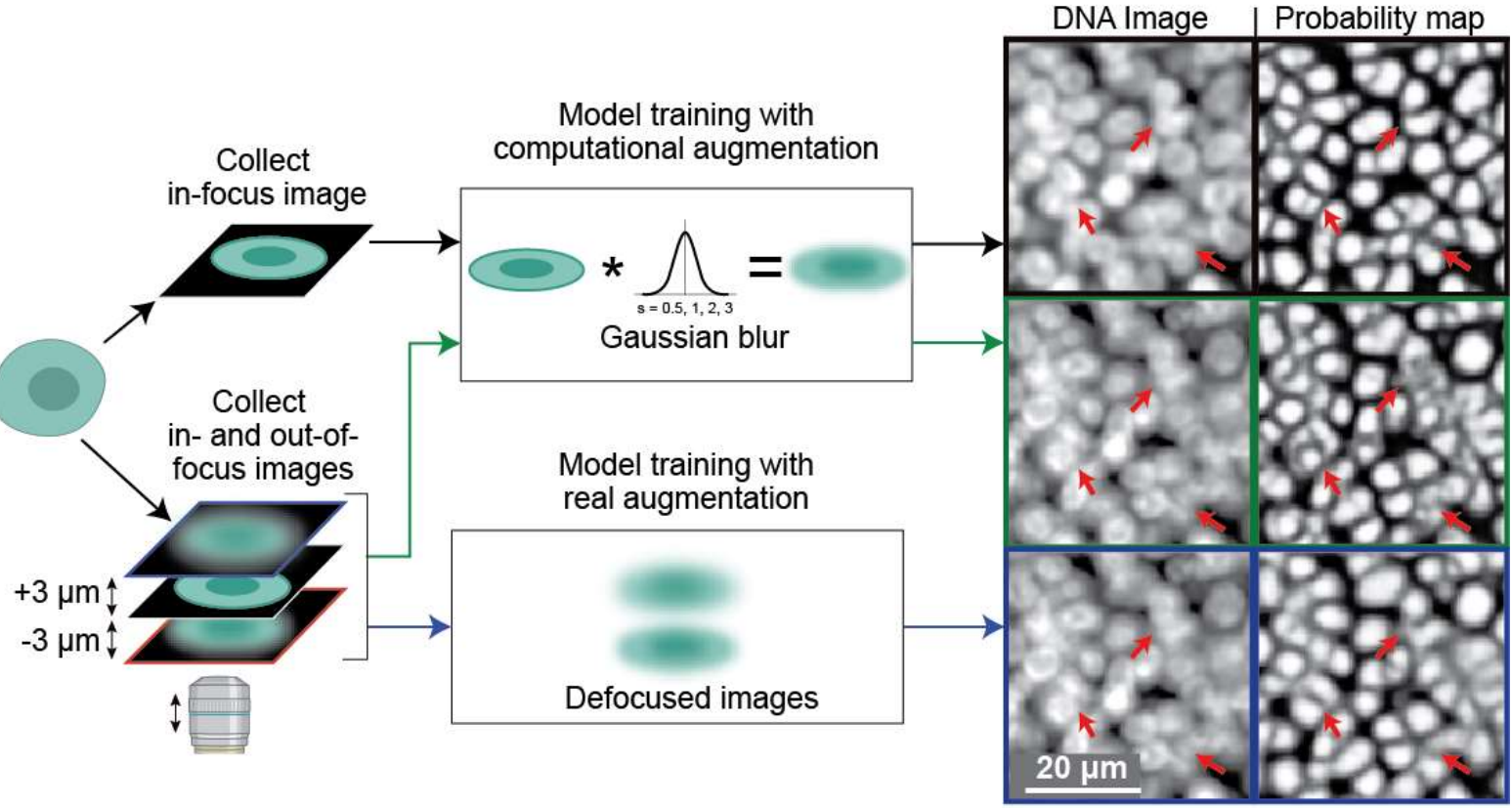

b

UnMICST-M

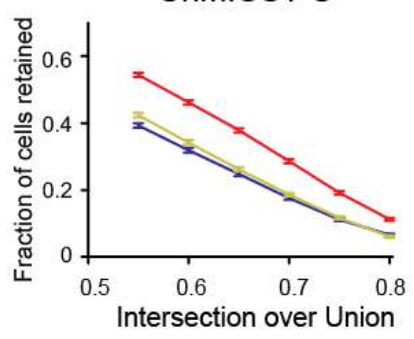

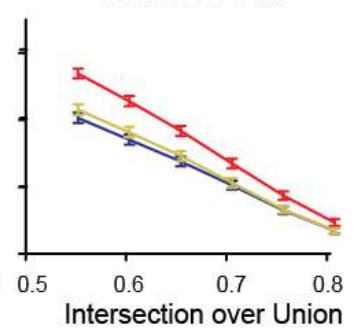

UnMICST-P

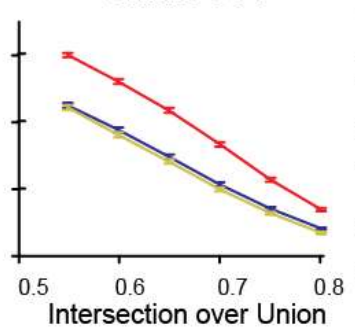

C

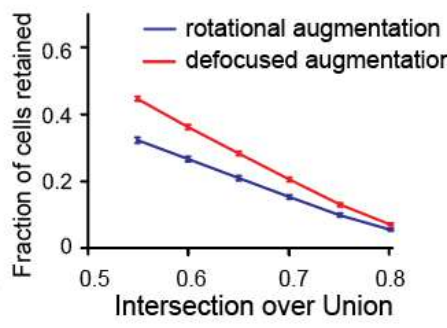

— no augmentation _ Gaussian blur — real augmentation

Figure 1: Comparing the use of real augmentations (defocused and overexposed images)

and Gaussian blur. a) Schematic diagram showing the approach comparing test images on models trained with Gaussian-blurred or defocused image data. Higher contrast probability maps signify more confidence - areas of interest are highlighted with red arrows. Corresponding probability maps indicate a model trained with defocused images performs better on defocused test images than a Gaussian-blurred model. Scale bar denotes 20 micrometers. b) Plots show that incorporating real augmentations (red curve) into the training set is statistically significantly superior to training sets with Gaussian blur (yellow curve) and without real augmentations (blue curve) for UnMICST-U, UnMICST-M, and UnMICST-P. Simulating defocused images with Gaussian blur is only marginally better than not augmenting the training data at all. c) Comparing UnMICST-U model accuracy when the training dataset size was held constant by replacing defocused augmentations (red curve) with 90 and 180 degree rotations (blue curve). 


\section{Figure 2}

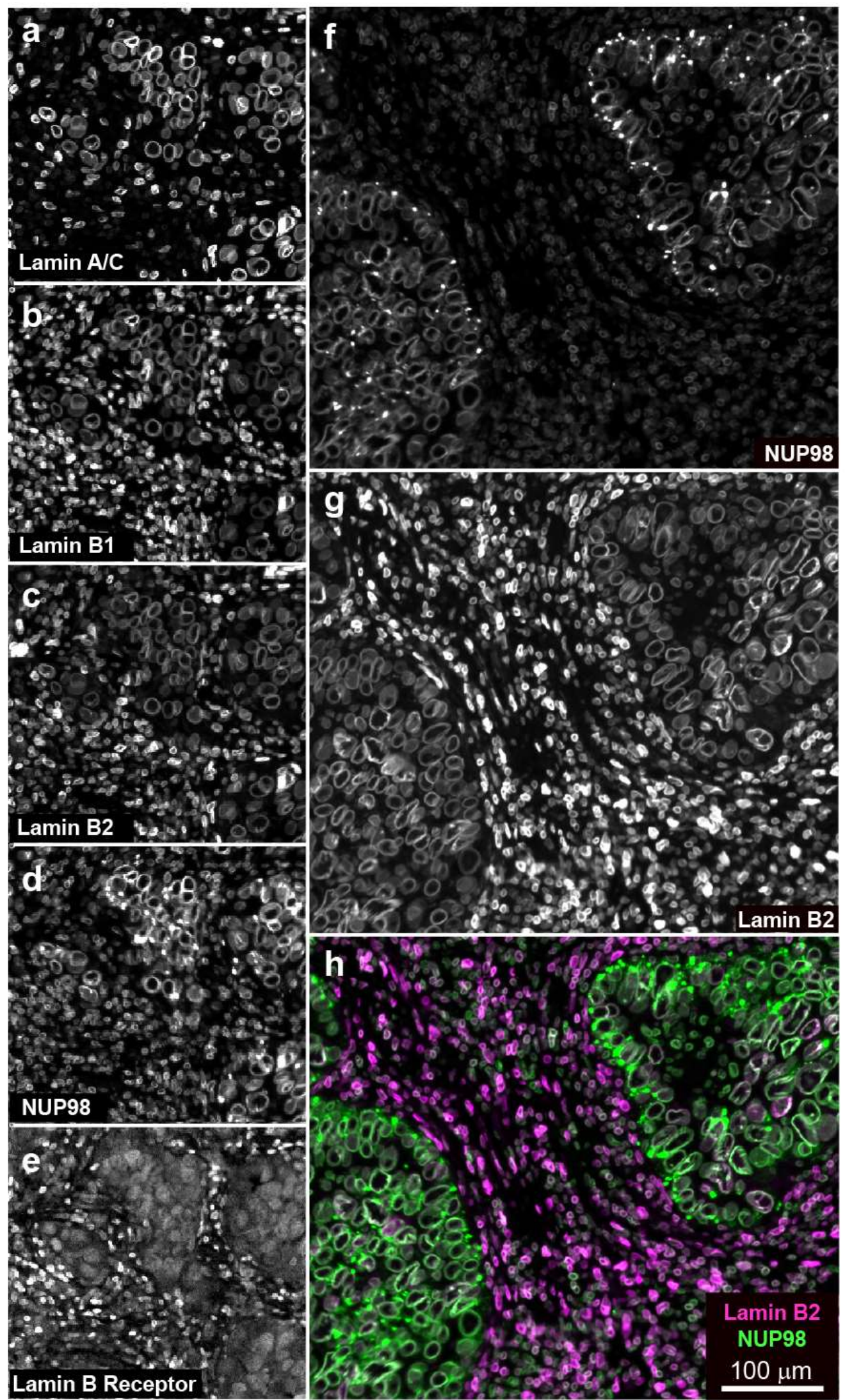




\section{Figure 2: Comparing different nuclear envelope stains in colon adenocarcinoma. a-e)}

712 Showcasing lamin A/C, lamin B1, lamin B2, NUP98, and the lamin B receptor in the same field of 713 view. Lamin B1 and B2 appear to stain similar proportions of nuclei while lamin A/C stains fewer

714 nuclei. The stain against the lamin B receptor was comparatively weaker. Lamin B2 (f) and NUP98

715 (g) are complementary and, when used in combination, maximize the number of cells stained. $\mathbf{h}$ )

716 Composite of lamin B2 (purple) and NUP98 (green). Scale bar denotes 100 micrometers. 


\section{Figure 3}

a

Colon

Small Int.

Tonsil

Lung

Fibroblast

GBM
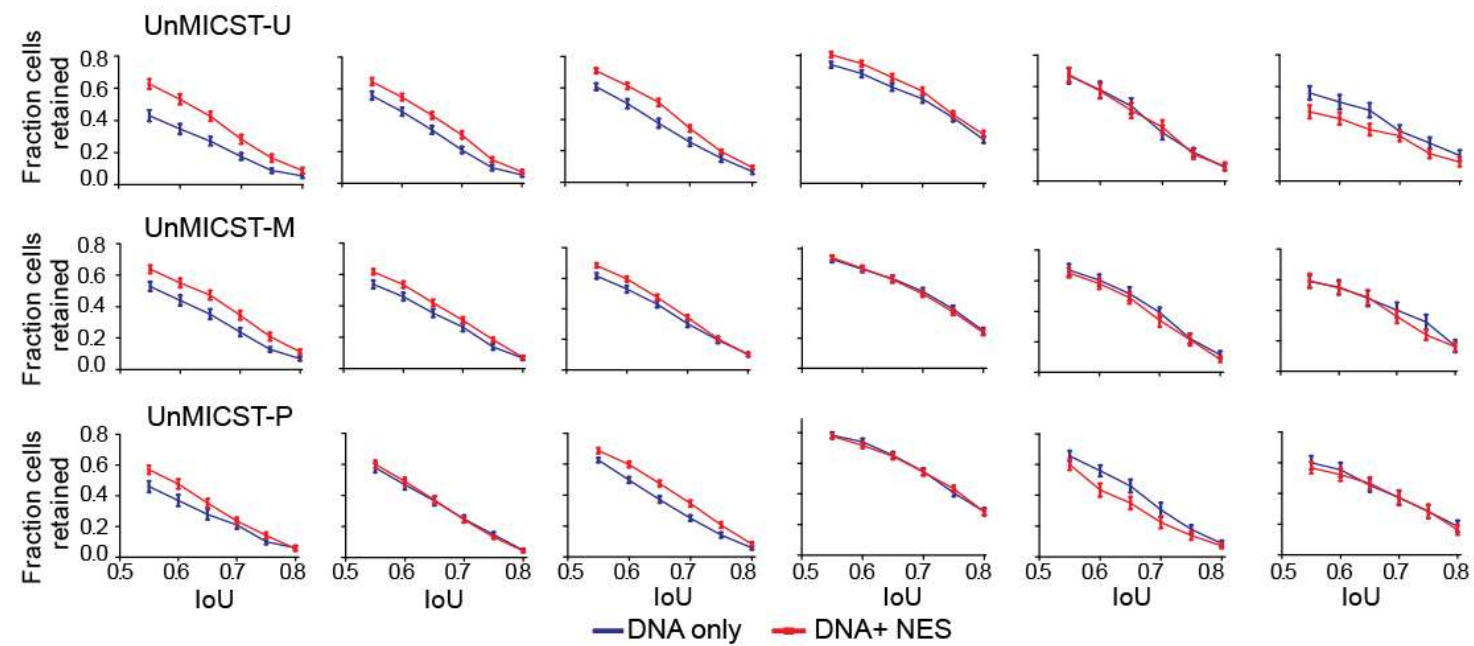

b

Colon

Small Int.

Tonsil

Lung

Fibroblasts

GBM
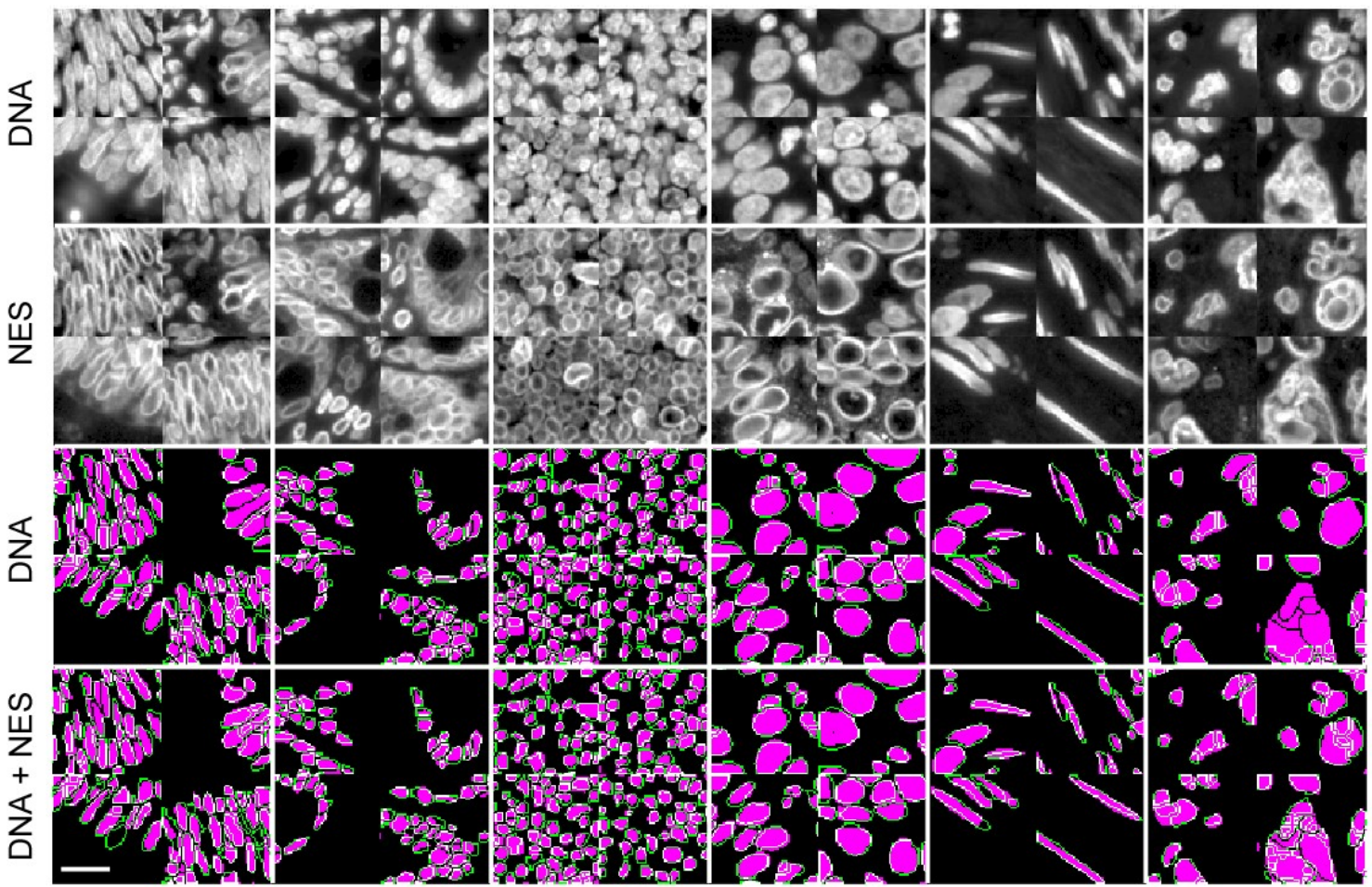

Prediction Ground truth

Figure 3: NES with DNA improves nuclear segmentation. NES - nuclear envelope staining. Assessing the addition of NES as a $2^{\text {nd }}$ marker to DNA on segmentation accuracy on a per tissue and per model basis. a) Variable loU plots comparing the DNA-only model (blue curve) and the DNA + NES model (red curve) across frameworks. Adding NES increased accuracy for densely packed nuclei such as colon, small intestine, tonsil, and to some extent, lung tissue. Error bars are 
723 standard errors of mean. b) Representative grayscale images of tissues stained with DNA and NES

724 comparing their variable morphologies, followed by UnMICST-U mask predictions (green) overlaid

725 onto ground truth annotations (purple). In tissue with sparse nuclei, such as fibroblasts from prostate

726 tissue, NES did not add an additional benefit to DNA alone. In tissues where NES does not exhibit

727 the characteristic nuclear ring, as in glioblastoma, the accuracy was similarly not improved. Scale

728 bar denotes 20 micrometers.

729

730

731

732 


\section{Figure 4}

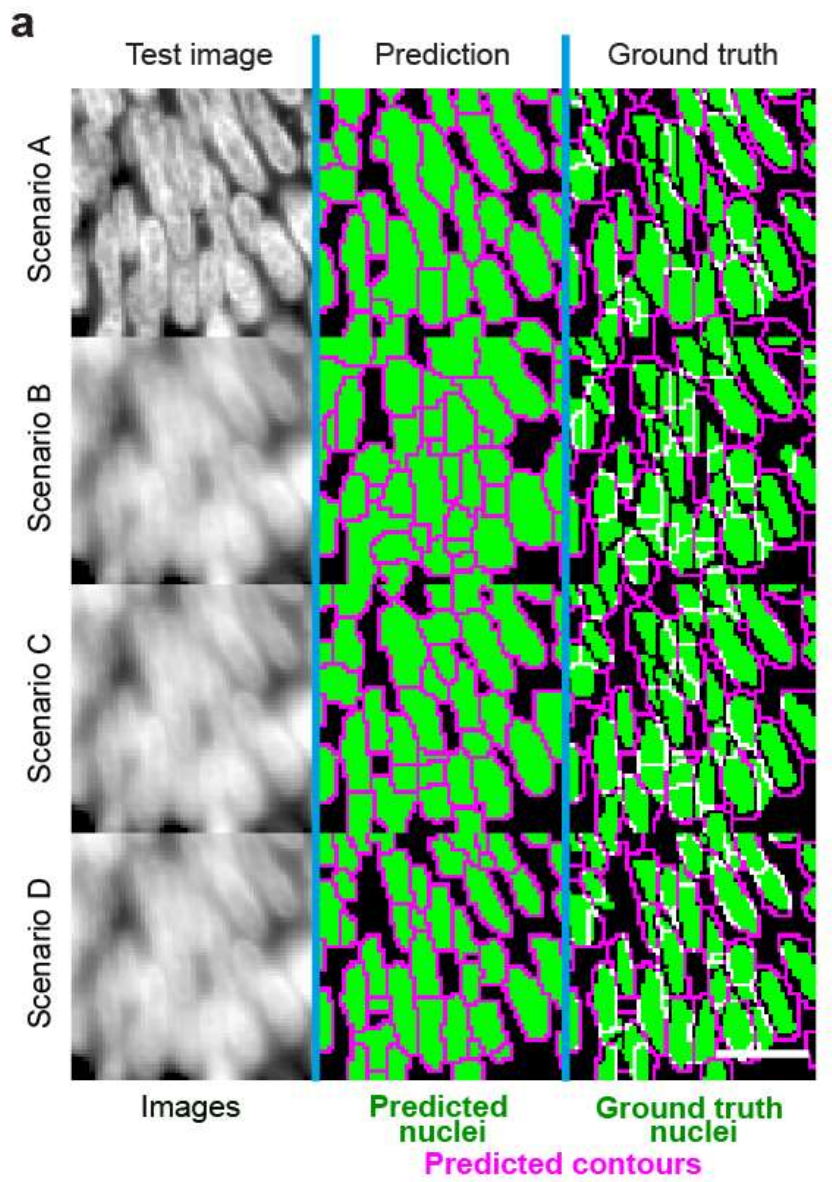

\begin{tabular}{|c|c|c|c|c|}
\hline Scenario & DNA & NES & $\begin{array}{c}\text { Real } \\
\text { augmen- } \\
\text { tation }\end{array}$ & $\begin{array}{c}\text { Defocused } \\
\text { test data }\end{array}$ \\
\hline A & Yes & No & No & No \\
\hline B & Yes & No & No & Yes \\
\hline C & Yes & Yes & No & Yes \\
\hline D & Yes & Yes & Yes & Yes \\
\hline
\end{tabular}

b
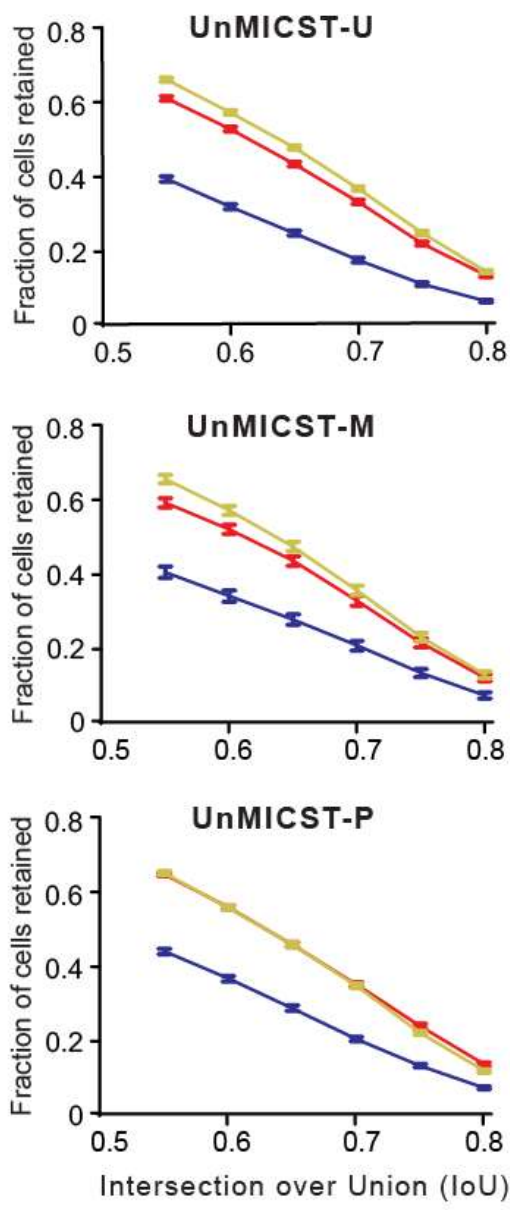

- DNA only, no augmentation

- DNA + NES, no augmentation

- DNA + NES + real augmentation

Figure 4: Combination of NES and real image augmentations on segmentation performance.

NES - nuclear envelope staining. a) Models trained with in-focus DNA data alone produced probability maps that were undersegmented, especially in densely-packed tissue such as small intestine (Scenario A). When tested on defocused data, nuclei borders were largely incorrect (Scenario B). Adding NES restored nuclei border shapes (Scenario C). Combining NES and real augmentations reduced false positive detections and produced nuclei masks better resembling the ground truth labels (Scenario D). Scalebar denotes 20 micrometers. Table legend shows conditions used for each scenario A-D. b) Graphs compare the accuracy represented as the number of cells 
743 retained across varying loU thresholds with all models from UnMICST-U (top), UnMICST-M

744 (center), and UnMICST-P (bottom). In all models, more nuclei were retained when NES and real

745 augmentations were used together during training (yellow curves) compared to using NES without

746 real augmentations (red curves) or DNA alone (blue curves).

747

748

749

750

751

752

753

754 


\section{Figure 5}

a
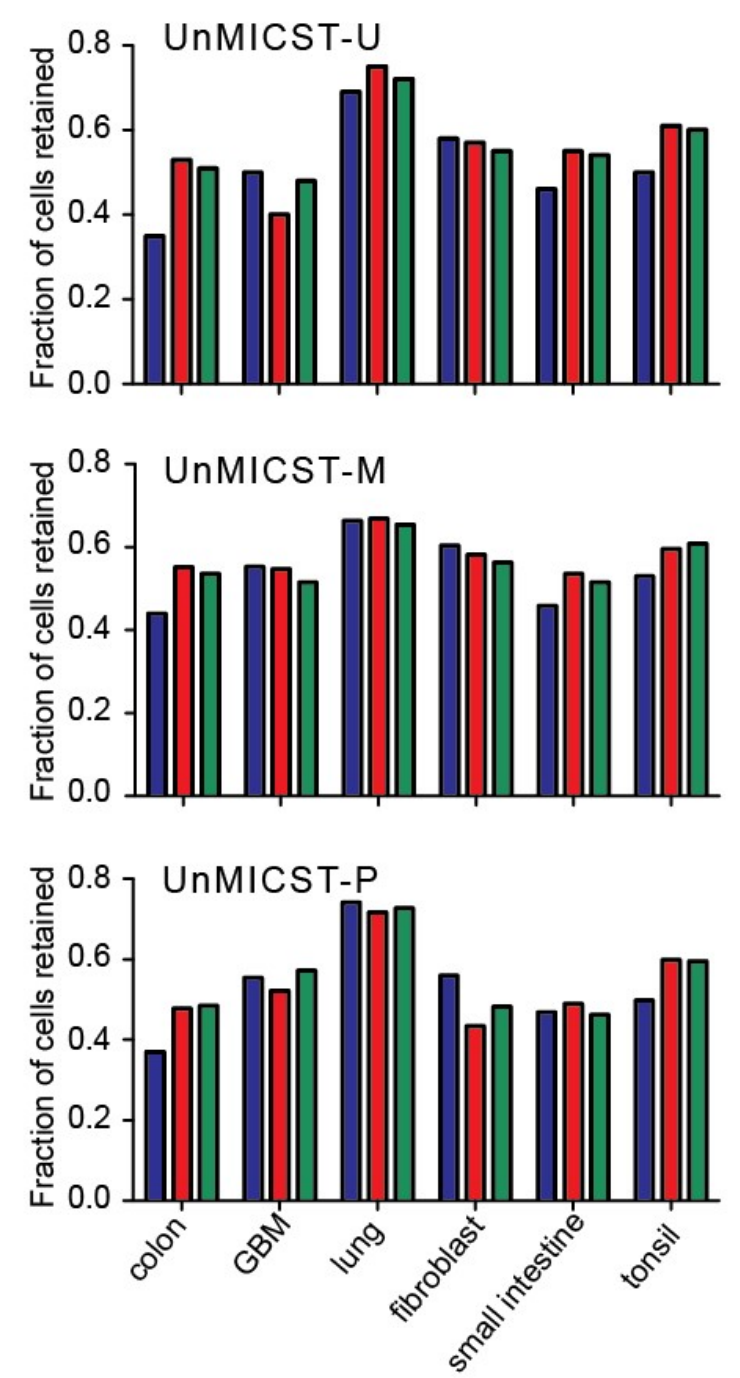

b
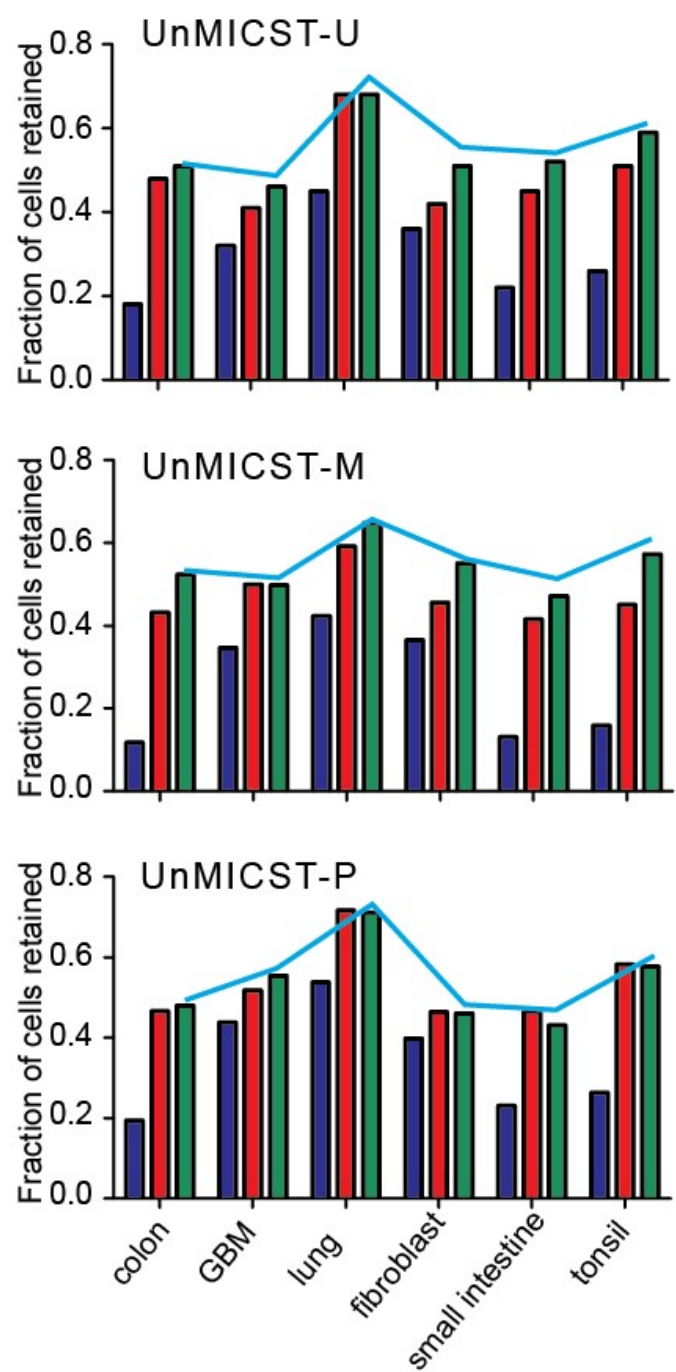

Figure 5: Assessing different training strategies on a) in-focus and b) defocused test data for different tissue types. a) In all tissue types apart from GBM, the addition of NES (red bars) and the use of real augmentations combined with NES (green bars) in training data offered superior accuracy compared to using DNA alone (blue bars). b) When the models were tested on defocused data, all tissues (including GBM unexpectedly) showed benefits resulting from using NES (red bars) combined with real augmentations (green bars). The line plot indicates highest accuracy achieved for each tissue when tested on in-focus data from panel a. 


\section{Figure 6}

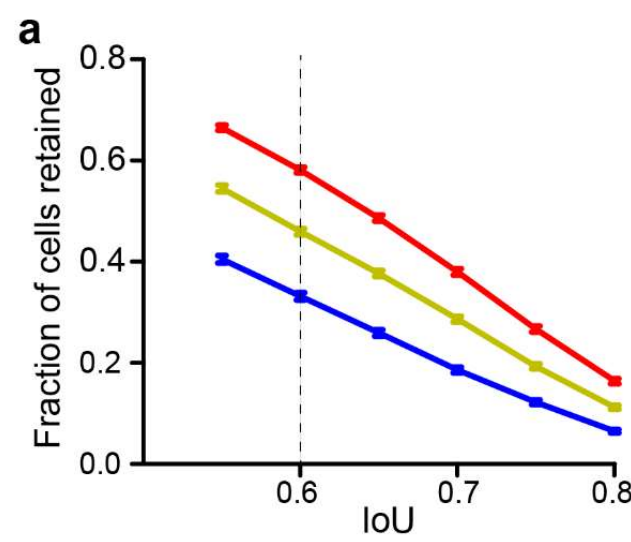

Training Data Augmentation

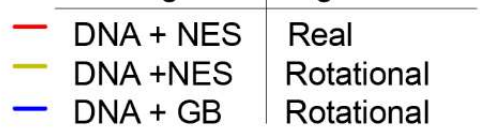

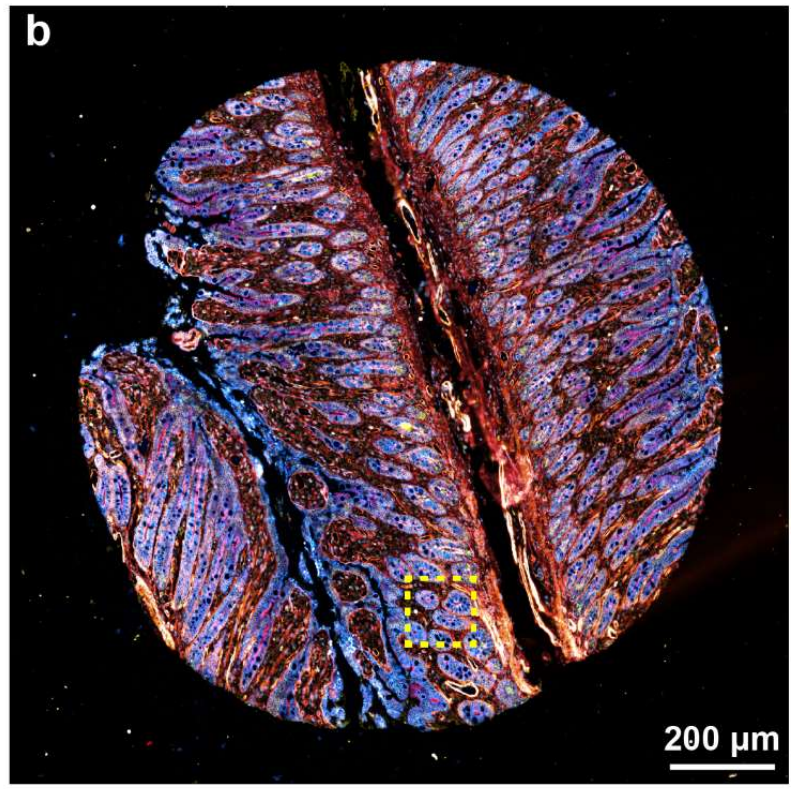

C

E-cadherin Levels

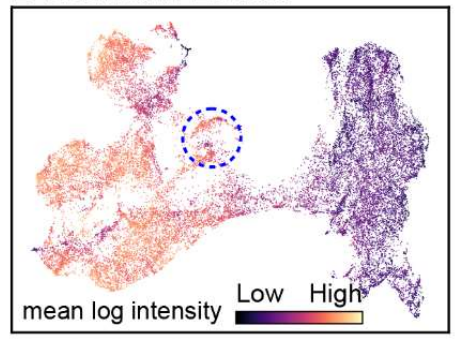

CD45 levels
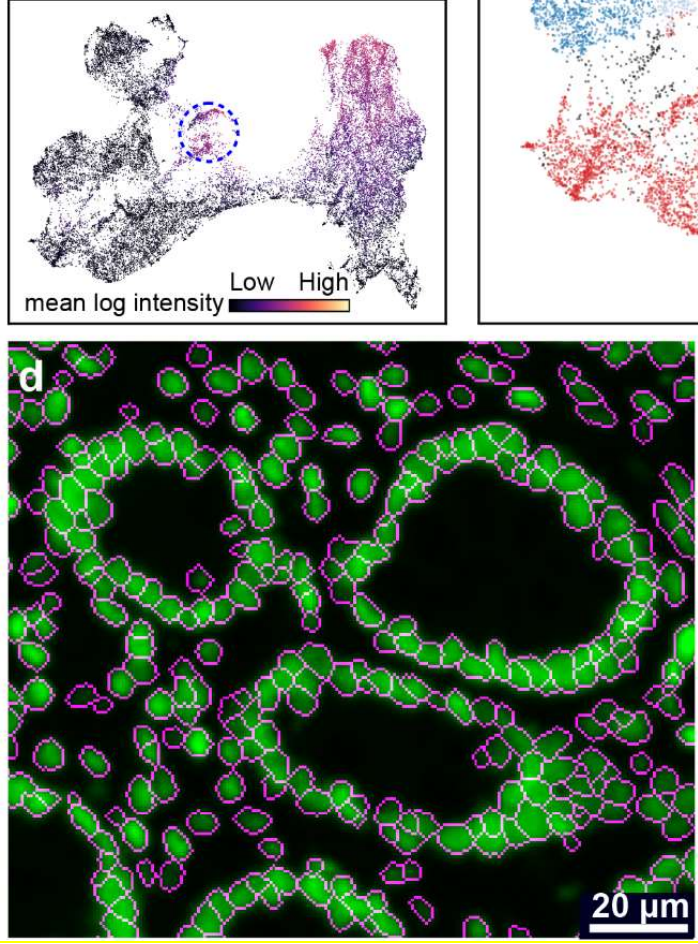

Density based clustering
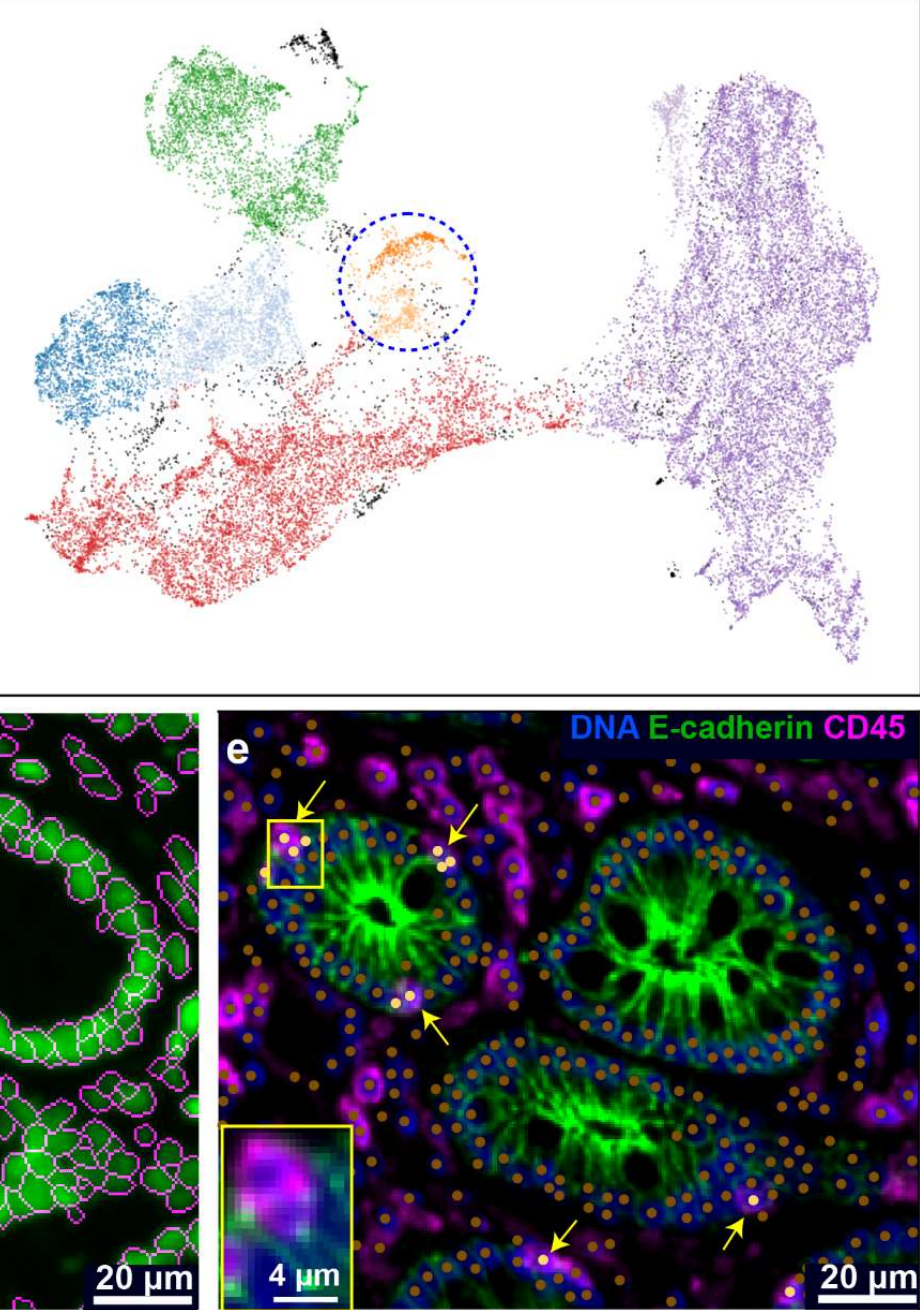


\section{Figure 6: Applying UnMICST models to highly multiplexed image data. a) Accuracy}

765 improvement of UnMICST models trained with and without NES (nuclear envelope staining) as

766 compared to DNA alone, and real augmentations as compared to computed blur (GB; Gaussian

767 blur). To balance training dataset size, GB was substituted for NES data and computed 90/180

768 degree rotations were substituted for real augmentations. b) A 64-plex CyCIF image of a non-

769 neoplastic small intestine TMA core from the EMIT dataset. Dashed box indicates region of interest

770 for panels $\mathbf{d}$ and e. c) UMAP projection using single cell staining intensities for 14 marker proteins

771 (see methods). The color of the data points represents the intensity of E-cadherin (top left) or CD45

772 (bottom left) across all segmented nuclei. Density-based clustering using HDBSCAN identified

773 distinct clusters (each denoted by a different color) that were positive for either E-cadherin or CD45

774 as well as a small number of double-positive cells (blue dashed circle). d) Enlarged region of yellow

775 dashed box from b showing segmentation mask outlines (magenta) overlayed onto DNA channel

776 (green). e) Composite image of DNA, E-cadherin, and CD45 of the same region. Nuclei centroids

777 from segmentation denoted by brown dots. Cells positive for both E-cadherin and CD45 (from blue

778 dashed circle in panel c are marked with yellow arrows and yellow dots. Inset: enlarged view of

779 boxed region showing overlapping immune and epithelial cells. 


\section{Supplementary Files}

This is a list of supplementary files associated with this preprint. Click to download.

- SupplementaryMaterials.pdf 